\title{
Modeling and Additive Manufacturing of a Missing Teeth Human Mandible
}

\author{
Kode Jaya Prakash, Balla Srinivasa Prasad
}

\begin{abstract}
In biomedical engineering, fiber-reinforced polymers play an important role in creating physical biological models of patients, which helps surgeons explain anatomical details to patients and perform trial operations. Replicating the precise three-dimensional (3D) structure of the human mandible is now a priority for rebuilding these bones for complete functional and aesthetic healing. Additional production methods and reverse engineering are required to achieve the design of a personalized device with precise shape and size. The main purpose of this research work is to develop a specially developed human mandibular biological model using the additive manufacturing method, FDM (Fused Deposition Melting). FDM is performed by loading a CAD model into a $3 D$ Printer. We present this method using the FDM (Fused Deposition Melting) method to generate a human mandible biological model. Data obtained from computed tomography (CT) with a resolution of 1 mm was converted to a $3 D$ model by computer aided design (CAD) using CT digital imaging and medical communication (DICOM) data. After the CAD model is built, it is converted to a stereolithography (*.STL) format and then processed by rapid prototyping techniques to create a physical anatomical model using $3 D$ printing. Converting two-dimensional data (2D) from computed tomography data to a $3 D$ model is an accurate guide to shaping bone grafts. The current approach can translate treatment plans directly into the surgical field. It is an important teaching tool for forming and fixing implants, helping to counsel patients.
\end{abstract}

Keywords : ABS (Acrylonitrile Butadiene Styrene), human mandible, DICOM, STL, Additive manufacturing, 3D printing, Dentistry.

\section{INTRODUCTION}

In the past decade, significant advances have been made in the use of new manufacturing technologies and new materials for prosthesis and tissue engineering. Acrylic jaw models are commonly used in teaching, training, and medical research [1]. Reconstruction of osteosarcoma lobes is the primary method for treating sputum and facial defects after wasting half of the tumor or traumatic injury. In the absence of a model, the plate is bent in the usual way to accommodate the native mandible [2]. Rapid Prototyping (RP) is a layer-by-layer deposition technique that began in the 1980s. It greatly enhances the ability to create physical models with precise geometries using CAD (computer) design or data technology [3]. Production Additive
Technology (AM) was originally developed for industrial rapid prototyping in the late $1980 \mathrm{~s}$. A key feature of these is that the generation of physical objects is performed in a fully automated process based on a computer three-dimensional (3D) model. In addition, the use of a sintering strategy allows for the creation of very complex components in a matter of hours [4]. Because they allow you to create scanned data, direct access to anatomical parts such as image computed tomography (CT), in a complex way in medical RP medical images is useful. All of these models perform precise preoperative design to provide better illustration of human anatomy, in a variety of [5] surgical planning aids for surgeons and medical students with severe and practical applications. The RP model can also be used to design prostheses, prostheses and individual functions as a communication tool between the surgeon and the patient. The STL (Standard Tessellation Language) file format is required for this process. The "Cutter Software" will change the $3 \mathrm{D}$ model in a series of thin layers and give the code $\mathrm{G}$ code for the 3D printer to be processed in the STL file [6] The following layers are divided into a series of cross sections. In turn, all of these layers ensure that the 3D model is entered into the printer [7]. These layers are connected by a printer on the bed to create the $3 \mathrm{D}$ model needed for CAD-based design. In this method, an additive manufacturing method is used to manufacture a dental model. The program requires weekly model preparation to test and position the implant, especially for patients with missing teeth. However, when additives are used, the entire process is completed in one day.

\section{PROPOSED METHODOLOGY}

Methodology adopted in the present work is shown in figure 1. It consists of obtaining digital 3D patient data from CBCT (cone beam computed tomography) scan, most of the CBCT scans will generate output data in DICOM file format (Digital Communication in Medicine). DICOM file data (digital communication in medicine) needs to be imported into the Invesalius a medical modeling software to segment the DICOM image and ultimately convert the scanned CBCT data from DICOM to 'STL' (stereo lithography). Later errors in '.STL' are eliminated by using Netfabb software. Error free '.STL' is fed to a 3D printer (Makerbot Replicator $Z$ 18) for mandible printing. Supports formed during 3D printing will be removed before fixing to patient.

Kode Jaya Prakash, Research Scholar, Mechanical Engineering Department, GIT, GITAM University, Visakhapatnam, India.

Dr. Balla Srinivasa Prasad, Associate Professor, Mechanical Engineering Department, GIT, GITAM University, Visakhapatnam, India. 


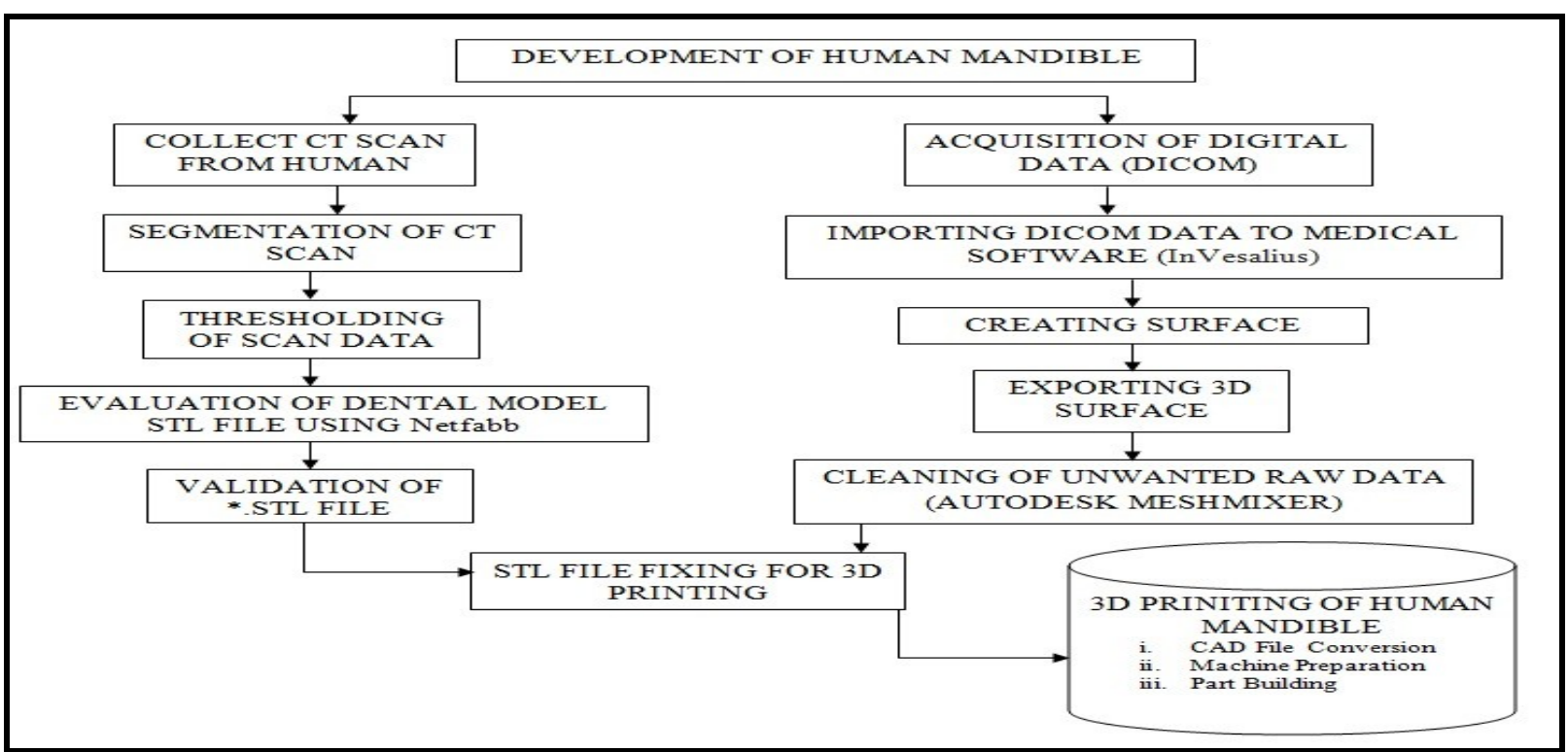

Figure 1. Proposed methodology in the present work

\subsection{PERFORMING A CT SCAN}

A 3D digital image is obtained using a CBCT (Cone Beam Computed Tomography) scanner. These imaging techniques are used to simulate the internal structure of the human body. The medical model processed by this data must be very accurate, so a helical scanning technique is needed to allow the complete volume scanning. Thus this will always allow to generate a huge number of sectors, and very importantly, the pixel size in each sector can be reduced accordingly. Most CT and MRI units have the ability to export the data in common a medical file format that is - DICOM - digital imaging and communication in medicine. Morphological data of the teeth was obtained using a CBCT scanner. A 3D data set will be obtained which produced 549 sagittal slices and with a thickness of $0.2-0.5 \mathrm{~mm}$ and was recorded in a medical digital imaging and communication (DICOM) file format, as shown in figure 2. CT scanned data is shown in the form different slices in figure 3 .

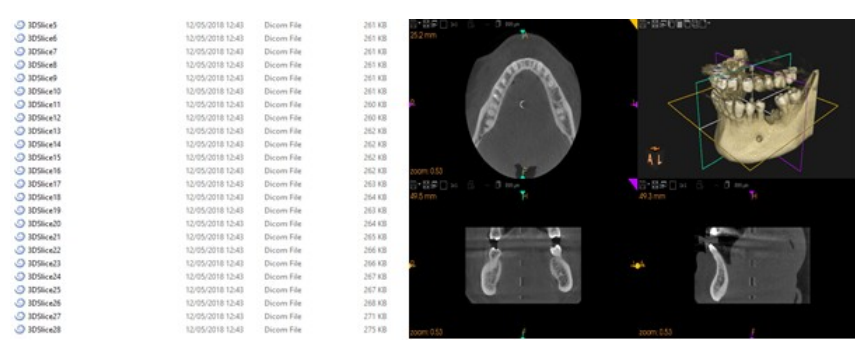

Figure 2. CT scan data of human mandible (DICOM SET)

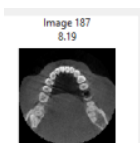

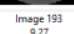

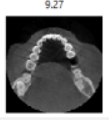

$\underset{\substack{\text { Image } 199 \\ 10.35}}{1.59}$

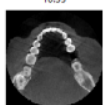

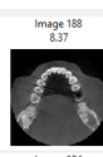
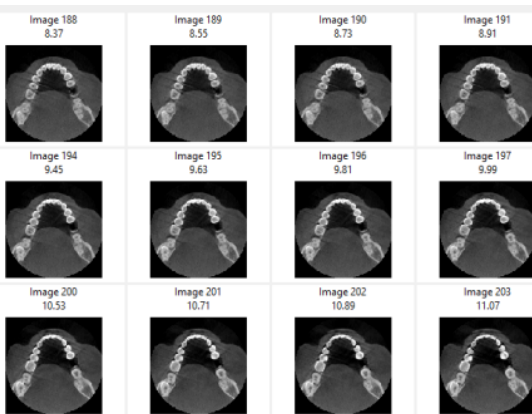

Figure 3. Different Slices of Scan Data

\subsection{DIGITAL DATA TRANSFER TO MEDICAL SOFTWARE}

The CT scan images in DICOM format are imported in InVesalius medical software and it is presented in figure 4. Medical software InVesalius is used to generate virtual reconstructions of the anatomy.

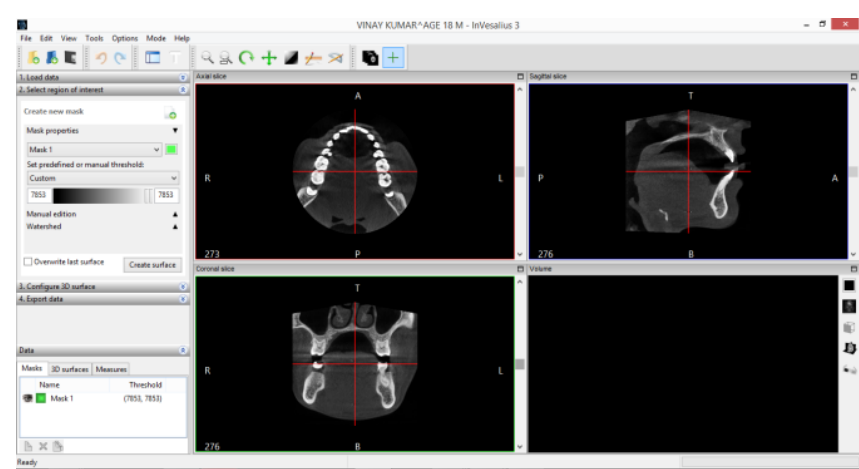

Figure 4. Importing Data CT scan data to InVesalius Medical Software

It is based on a two-dimensional image obtained by computed tomography, the software will generates a three-dimensional virtual model corresponding to the anatomy of the human body. After building a three-dimensional DICOM image, the software allows the generation of STL files (stereo lithography). InVesalius software has been developed to promote social integration of patients with severe facial deformities. The images of that particular contrast shown in the different views may be adjusted.

\subsection{SEGMENTATION AND THRESHOLDING OF CT SCAN}

The scanned CT data contains information about the region of interest and details of neighboring parts. Therefore, it is necessary to extract useful information from the scanned data. . The image correlation technique is a segmentation technique, and a segmentation technique including selecting a region of interest and generating a threshold image (gray quality) to provide a clearer representation of the region of interest may be used.

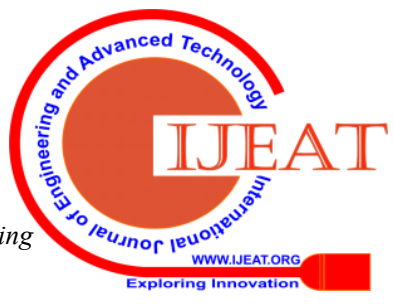


Segmentation of CT scan data is shown in figure 5 . Threshold is introduced in various proportions. This ensures that all substances consisting of bones or teeth are divided into masks. As a result, the hard tissue can be separated from the soft tissue of the face and separated from the hard tissue and the bony tissues into the mouth of the patient. Thresholding of Scan Data is presented in figure 6 .

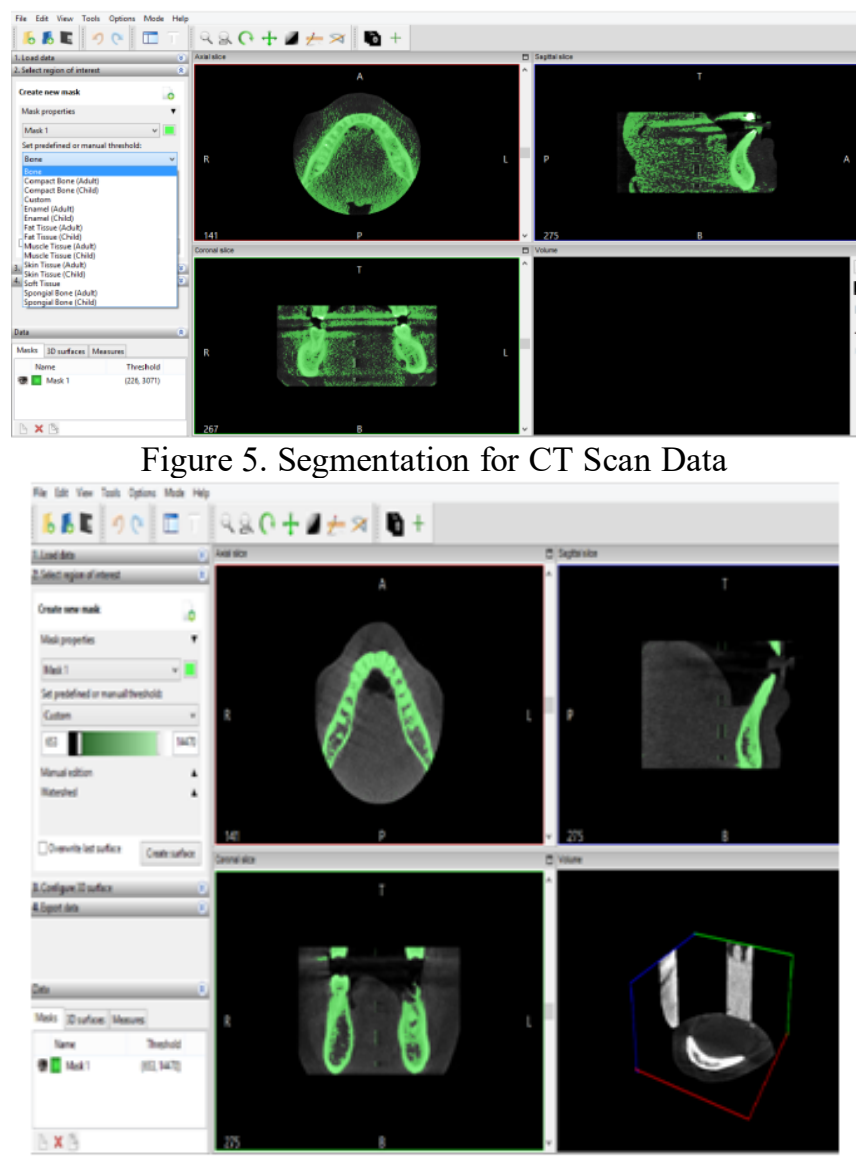

Figure 6.Thresholding of Scan Data

\subsection{CREATING AND EXPORTING 3D SURFACE}

After the threshold is set in a given area, a $2 \mathrm{D}$ image along with $3 \mathrm{D}$ image is obtained. The fourth layer in figure 7 (bottom right) shows the $3 \mathrm{D}$ view of the area selected by the threshold portion.

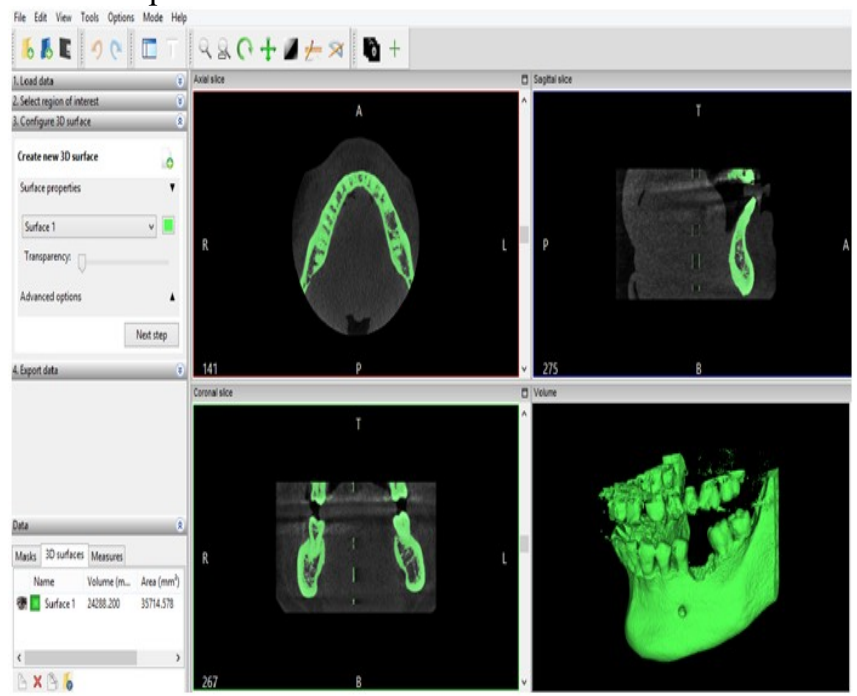

Figure 7. 3D surface model of the human mandible from the CT scan images

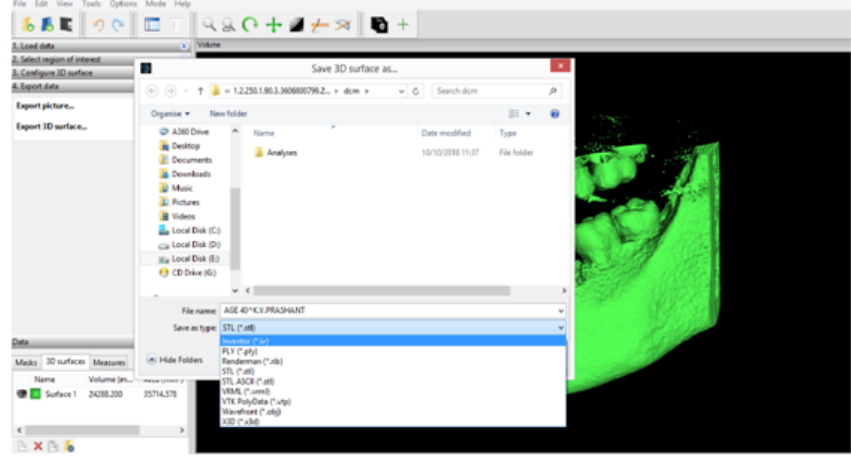

Figure 8.Exporting the data into *.STL file format.

After that, 3D surface exported and data file is saved with *.STL format. This STL file format is one of the standard file format for all rapid prototyping (RP) Systems is shown in figure 8.

\subsection{CLEANING OF UNWANTED RAW DATA USING AUTODESK MESHMIXER}

The Mesh mixer software is used to correct STL errors in the STL file and prepares the file for 3D printing. The imported dental model in Autodesk meshmixer is given in figure 9. The lower jaw of the mandible is the part to be printed; because of this the upper jaw should be removed. The upper jaw of the tooth model is removed by selecting a planar cutting operation as presented in figure 10 . Whereas, figure 11 shows the further noise elimination from lower jaw of the mandible to be aimed for 3D printing.
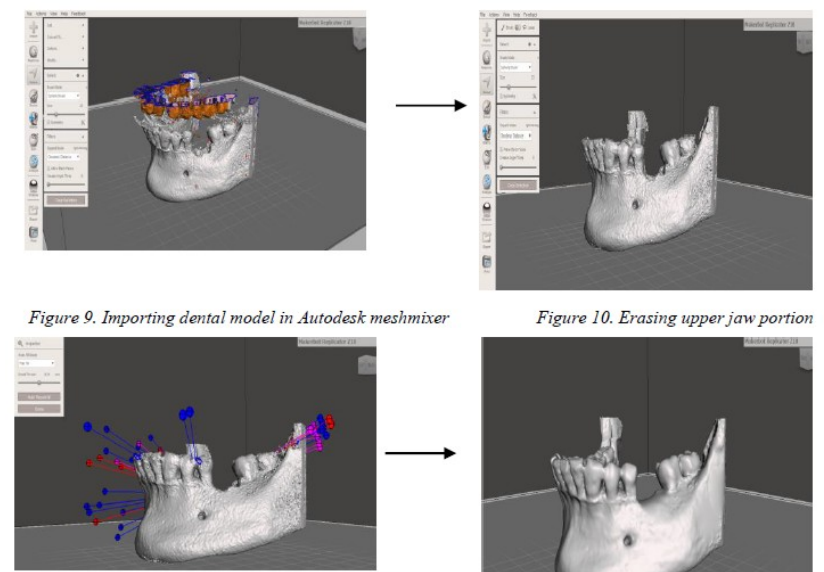

Figure 10. Erasing upper jaw portion

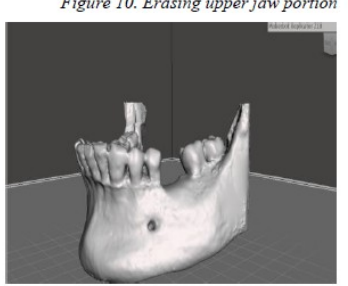

Figure 11. Elimination of noise matter \& small errors

2.6 EVALUATION OF *.STL DENTAL MODEL FILE USING NETFABB SOFTWARE

It is necessary to evaluate the model, quality and possible error verification before final printout. The created 3D model is used directly as input data (biological model) for the RP machine. Netfabb is a commercial software program, developed by Autodesk systems. After creating a 3D model, errors that prevent proper printing may occur. Openings in the grid, normal inversion, irrelevant edges and intersecting surfaces may cause printing errors. Increase the printability of models on 3D print exchange printers, Netfabb software is used. The software automatically detects error present in the STL file if any and also repairs the file to print the part smoothly without any interruption. Figure 12 
presents the validation of STL file using Netfabb console.

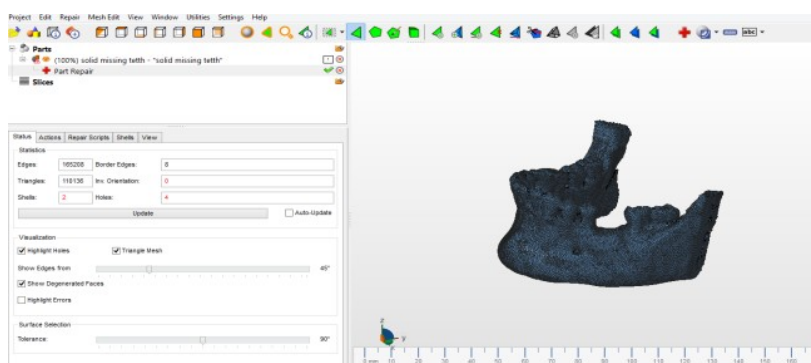

Figure 12. Validation of *.STL file using Netfabb console

The DICOM (Digital Communication in Medicine) images are segmented to construct a 3D Model in stereo lithography format (STL format). The CAD model which is converted to *.STL file format represented as surface made of triangles. While generating the $3 \mathrm{D}$ reconstruction it is termed as Voxels (3D equivalent of a pixel) and it lies between the image slices and with using a selected value for the variation of density intensity. Voxel corners of the 3D model were thoroughly examined. The $3 \mathrm{D}$ models which is acquired form the medical data contains lot of errors. The errors include holes, unfilled gaps and cracks which is termed as STL errors. Before printing a 3D model errors from the STL file has to be rectified.

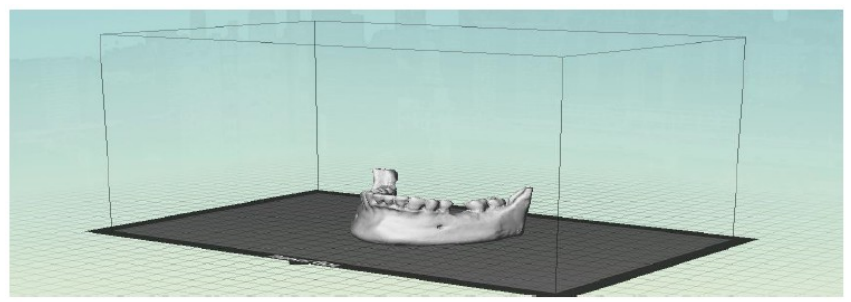

Figure 13. Orientations of mandible model in Makerbot Maker ware

For correcting the STL files again it has to be processed in a STL correction software. In this present work for repairing STL file the software used is Netfabb. Later, Makerbot Desktop Beta software is used to print parts from the generated 3D CAD model via the Makerbot 3D printer is shown in figure 13.

\section{MANUFACTURING PROCEDURE}

\subsection{CAD File Conversion}

The CAD file will be imported in STL format. The Makerbot Maker ware software divides the model into a series of layers in which the 3D part to be printed will be examined. These cutting voices create the moment of the extruder nozzle moving in different $\mathrm{X}, \mathrm{Y}$ and $\mathrm{Z}$ directions.

\subsection{Machine Preparation}

MakerBot Replicator 2X Desktop 3D printer and its specifications are presented in figure 14. 3D printer must maintain optimal temperatures. The temperature that will be maintained for the construction chamber and the nozzle is set to be between $110^{\circ} \mathrm{C}$ and $2300 \mathrm{C}$.

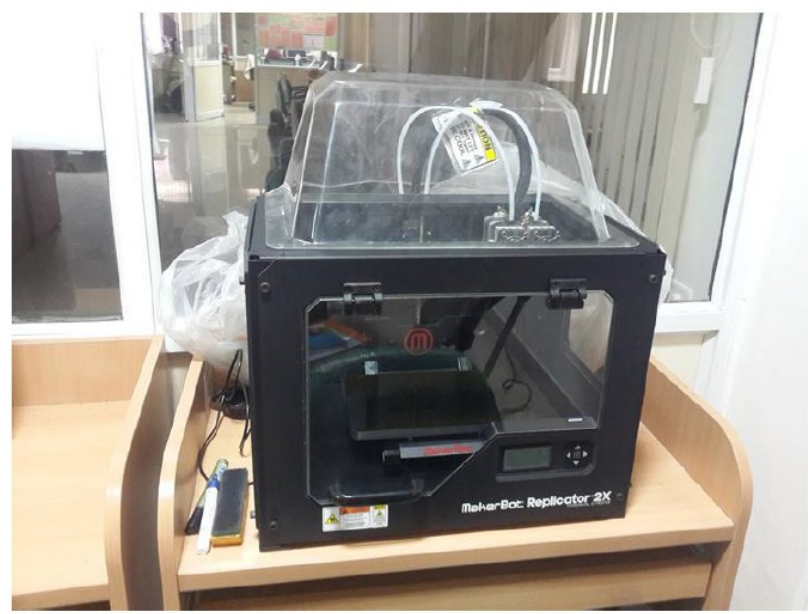

Figure 14. Makerbot Replicator 2X - 3D printer

\subsection{Part Building}

While the material is about to be extruded from the nozzle according to the CAD design in figure 15, the $\mathrm{Z}$ Stage platform rises to its initial position, so the extruder starts to build the part that moves in the X, Y direction. After the first layer is completed, the $\mathrm{Z}$ phase construction platform move down and allows the next level to continue in the previous level. So this process continues until the full part is created. Properties of materials used for printing human mandible are given in "table 1 ".

Table 1. Print statistics

\begin{tabular}{|l|l|}
\hline \multicolumn{2}{|c|}{ 3D Printed dental model statistics } \\
\hline Material type & ABS \\
\hline Layer height(mm) & 0.2 \\
\hline Number of layers & 222 \\
\hline Number of shells & 2 \\
\hline Infill (\%) & 50 \\
\hline Build time & $2 \mathrm{~h} 54 \mathrm{~min}$ \\
\hline Filament used (grams) & 40.10 \\
\hline
\end{tabular}

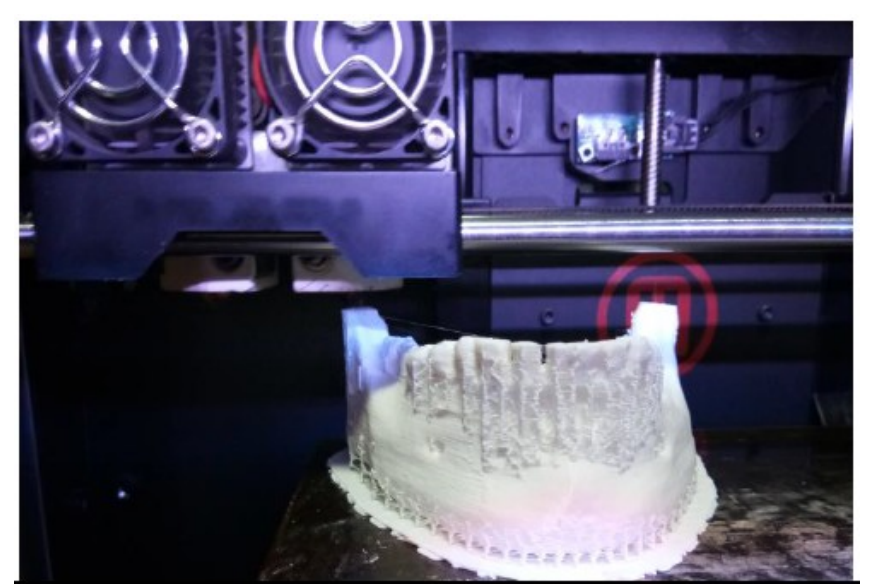

Figure 15. Human mandible with support structures while $3 \mathrm{D}$ printing

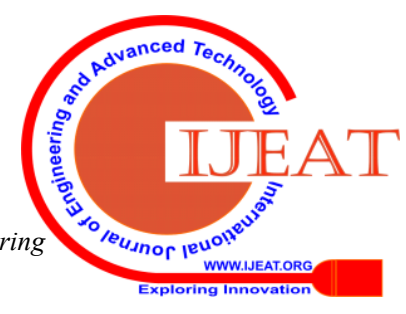




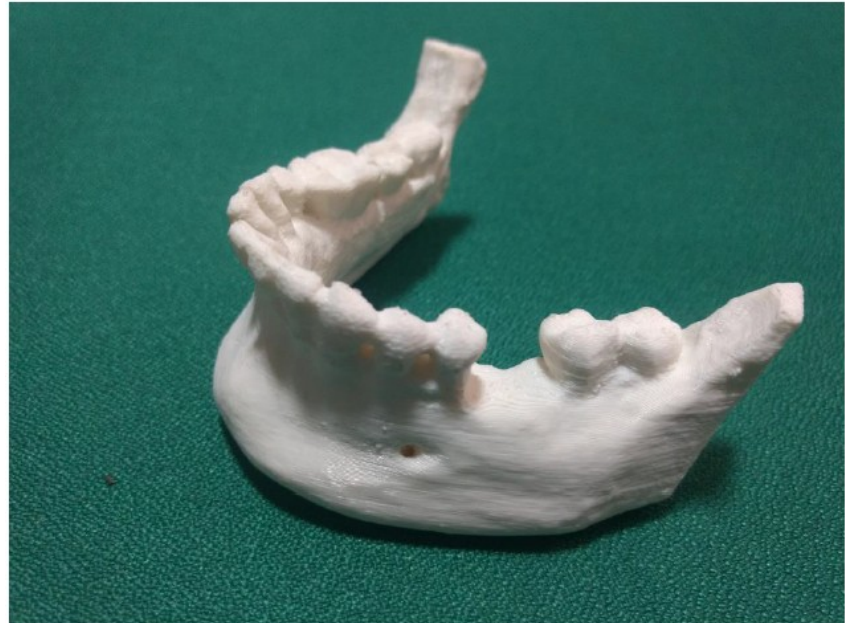

Figure 16. 3D Printed human mandible with missing teeth after removing support structures

Finally, 3D printed human mandible after removing support structures is presented in figure 16 and this mandible is ready for replacement to the patient.

\section{CONCLUSION}

Conventional manufacturing of the dental models are time taking task and they are purely depends on the dental technician skills and they are not that much accurate in case of patient specific. In order to overcome these problems digital manufacturing has came into existence. In the present work dental model is printed by using the CBCT Scanned data of the human jaw from the person aged about 43 years. Invesalius medical modeling software is used for extracting the medical data in the form of DICOM Format to STL format. Post processing of the scanned raw data is highly necessary before printing of the bio model. The post processed STL file is used for 3D printing of the bio- model of the jaw by using Makerbot replicator 2x. To perform the surgery real time models are necessary for the dental doctors to improve the success rates of the surgery. So physical models are quite attractive to hold the model in the hand and provides in detail anatomy from patient to patient very accurately. The surgeon and the patient communication will be improved by the use of the physical models before perform the complex surgeries especially while doing the implantations.

\section{ACKNOWLEDGEMENTS}

The authors sincerely acknowledge the support of the VNR Vignana Jyothi Institute of Engineering \& Technology, Hyderabad, India for providing the 3D Printing facility at Mechanical Engineering Department to carry out the research work.

\section{REFERENCES}

1. Martorelli, M., Maietta, S., Gloria, A., De Santis, R., Pei, E. and Lanzotti, A., 2016. Design and analysis of 3D customized models of a human mandible. Procedia CIRP, 49, pp.199-202.

2. Kumta, S., Kumta, M., Jain, L., Purohit, S. and Ummul, R., 2015. A novel 3D template for mandible and maxilla reconstruction: Rapid prototyping using stereolithography. Indian Journal of Plastic Surgery: Official Publication of the Association of Plastic Surgeons of India, 48(3), p.263.
3. Peltola, S.M., Melchels, F.P., Grijpma, D.W. and Kellomäki, M., 2008. A review of rapid prototyping techniques for tissue engineering purposes. Annals of medicine, 40(4), pp.268-280.

4. Levy, G.N., Schindel, R. and Kruth, J.P., 2003. Rapid manufacturing and rapid tooling with layer manufacturing (LM) technologies, state of the art and future perspectives. CIRP Annals-Manufacturing Technology, 52(2), pp.589-609.

5. Kontio, R., Björkstrand, R., Salmi, M., Paloheimo, M., Paloheimo, K.S., Tuomi, J. and Mäkitie, A., 2012. Designing and additive manufacturing a prototype for a novel instrument for mandible fracture reduction. Surgery S, 1, pp.2161-1076.

6. Parthasarathy, J., 2014. 3D modeling, custom implants and its future perspectives in craniofacial surgery. Annals of maxillofacial surgery, 4(1), p.9.

7. Chua, C.K. and Leong, K.F., 2014. 3D Printing and Additive Manufacturing: Principles and Applications (with Companion Media Pack) of Rapid Prototyping Fourth Edition. World Scientific Publishing Company. 